\title{
Visual cells in excised Limulus eyes: Dark adaptation reveals evidence of response duality
}

\author{
LOLIN T. WANG-BENNETT and GERALD S. WASSERMAN \\ Purdue University, West Lafayette, Indiana
}

\begin{abstract}
The role of light adaptation on response duality was examined in excised eyes taken from mature Limulus and maintained in ordinary seawater for up to $10 \mathrm{~h}$. Several manifestations of duality were found. Two types of quantum fluctuations exist in dark adaptation. Two segments were found in the intensity-response function characterizing the plateau phase of responses in the dark-adapted state. And this intensity-response function covered a wide range of intensities in dark adaptation. Light adaptation reversibly abolished all three of these expressions of response duality. Thus, despite repeated claims that response duality is a property that degenerates after excision, these data show that it is an adaptation-dependent property that can readily be seen when care is taken to allow enough time for dark adaptation.
\end{abstract}

The purpose of this note is to put on record some results regarding the viability of excised Limulus eyes because more complete accounts have not been published in the appropriate journals.

Excised horseshoe crab (Limulus polyphemus) eyes have long been useful for studying visual cell function, because this preparation readily supports intracellular recordings using glass microelectrodes from single cells. But most studies in which this preparation has been used have rarely lasted more than a few hours. Furthermore, excision is generally done under ordinary room illumination, and little time is usually allowed for dark adaptation. As a result, new properties have been discovered from time to time when Limulus eyes have been allowed to dark adapt more fully. An example was the discovery of quantum-evoked discrete potential fluctuations (Fuortes \& Yeandle, 1964) of two sizes (Adolph, 1964; Dowling, 1968).

Subsequently, Barlow and his co-workers (Bayer \& Barlow, 1978; Barlow \& Kaplan, 1971, 1977; Kaplan \& Barlow, 1975) reported that the larger discrete potential fluctuation can be substantially bigger than the smaller type, that a single photoreceptor cell can operate over a very wide range of light intensities, and that the wide range is achieved by means of an intensity-response function that has two segments, each operating in a different portion of the intensity range. These observations were taken as converging indications of dual response mechanisms in these cells.

Collection of these data was supported in part by a National Eye Institute Grant EY-07008 and in part by a David Ross Fellowship. Preparation of this report was supported by a National Science Foundation Grant EET 87-21333. Lolin T. Wang-Bennett's present affiliation is: Department of Otorhinolaryngology, Baylor College of Medicine, Houston, TX. Correspondence should be sent to Gerald S. Wasserman, Sensory Coding Laboratory, Department of Psychological Sciences, Purdue University, West Lafayette, IN 47907; e-mail: codelab@psych.purdue.edu.
Barlow and his co-workers did not, however, conclude that dark adaptation was the key variable in their observations, because they worked on cells resident in unexcised eyes of otherwise intact Limulus. These in situ observations, and the absence of such effects in excised eyes in vitro (although these phenomena were reported sometimes to survive for an unspecified short time after excision), led them to conclude that such phenomena could not be reliably seen in vitro. They believed that their conclusion was further supported by their finding that cardiac disruption abolished the new phenomena. Such disruption was also reported to reduce sensitivity by as much as five log units. It was concluded that excision in vitro or exsanguination of an in situ preparation progressively abolished a sensitive component of the normal visual mechanism, leaving a preparation that contained only "remnants" of the "natural depolarizing potential" (Barlow \& Kaplan, 1977, pp. 218-219).

However, the argument from exsanguination is not decisive, because there are actually two possibilities to be considered: (1) The loss of circulating blood could have deprived cells of vital support necessary to maintain the more sensitive component. (2) Mechanical or toxic effects associated with cardiac disruption could have damaged the cells from which the recordings were being taken.

In fact, the second possibility is more likely to be correct, because, as will be reported in detail here, we have repeatedly seen these phenomena in excised eyes (Wang \& Wasserman, 1980, 1984). However, we do not see them in freshly excised eyes; they only appear after some time. Instead of progressively disappearing, they progressively develop. Since cardiac support is long gone by the time we see these effects, it cannot be the case that cardiac support is required to maintain the function that underlies these phenomena.

We believe that we have seen them because our procedure differs from that used by most other investigators. 


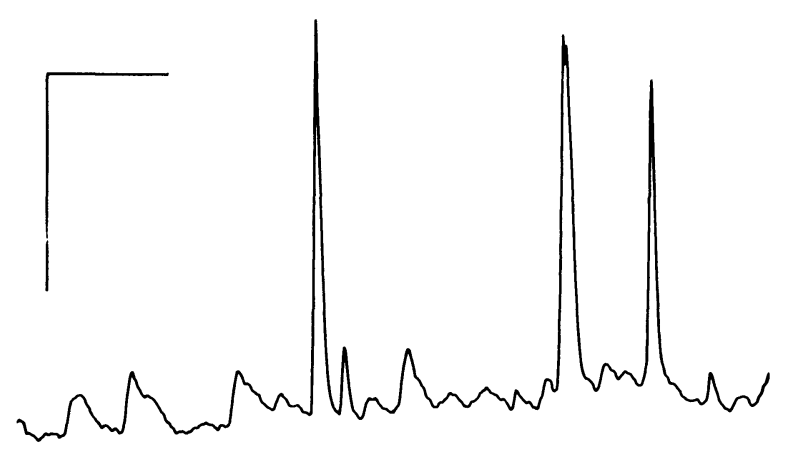

Figure 1. Transmembrane potential as a function of time in an intracellular recording taken from an excised Limulus lateral eye photoreceptor cell exposed to a continuous very $\operatorname{dim}$ light $\left(4 \times 10^{-6} \mathrm{~W} / \mathrm{m}^{2}\right)$ in a state of dark adaptation. Two types of potential fluctuations are seen: There are three large potential fluctuations that are about $20 \mathrm{mV}$ in amplitude, and there are over a dozen small potential fluctuations that are less than $5 \mathrm{mV}$ in amplitude. The calibration marker represents $10 \mathrm{mV}$ and 1 sec.

The difference is dictated by our interest in neural coding, which forces us to spend many hours recording from a particular cell and which also requires that the cell must remain in a constant state of sensitivity for hours. Experience has taught us that it is futile to begin an experiment in a freshly penetrated cell; many such cells steadily decline in sensitivity. Instead, we let a promising cell sit unstimulated in darkness for approximately an hour. When we do begin an experiment, we check the sensitivity of the cell by regularly delivering weak test flashes at half-minute intervals. This readily permits us to detect sensitivity changes much smaller than five log units. We can easily detect changes as small as a third of a log unit. We use this sensitivity check and an associated check of the stability of the membrane potential to discard cells that change in either characteristic by more than $5 \%$ under otherwise constant conditions. (Further details on our procedures and on the quality of the data have been given elsewhere; see Wang \& Wasserman, 1985.)

We use mature animals that measure 6-8 in. across the carapace. Our experiments generally last 5-10 h. We find that the excised visual cells that meet our stability criterion for such long periods characteristically exhibit the properties reported by Barlow and his co-workers. Obviously, a fair presumption is that it is precisely the same cells that were least damaged by the penetration of the microelectrode. As an example of the phenomena we routinely observe, Figure 1 shows a recording taken from an adult lateral eye, which had been excised for more than $5 \mathrm{~h}$ at the time of the recording. This recording contains a number of discrete potential fluctuations. Three fluctuations are quite large and average close to $20 \mathrm{mV}$ in amplitude, while the remainder are quite small and fall mostly below $5 \mathrm{mV}$. These two amplitude values quantitatively fall quite near the central tendencies of the amplitude distributions of data typically obtained in cells in intact eyes that have been held for a long enough period of time to obtain a statistical distribution (cf. Barlow \& Kaplan, 1977, Figure 2).

We emphasize that the large potential fluctuations were not evident immediately after penetration; they only appeared and then grew progressively larger as the cell became dark-adapted. They could be reversibly suppressed by light adaptation. We believe that the necessary condition for Barlow and his co-workers to have observed similar phenomena was that their intact eye experiments could and did last long enough to permit complete dark adaptation.

Adaptation also affects such cells' response range. Figure 2 shows photoreceptor potentials recorded in dark and light adaptation over a wide range of intensities in two states of adaptation. Adaptation was regulated by adjusting the intensity of a train of adapting flashes delivered prior to the delivery of the test flash. The responses to the test flashes are shown in Figure 2. They exhibit the usual properties found in visual cells: an initial transient followed (at higher intensities) by rapid self-adaptation in the form of a sag back to a steady-state plateau. The quantal nature of the response is most evident at lower intensities, where the discrete potential fluctuations are easily resolvable and produce a potential waveform that is quite irregular. At higher intensities, the number of discrete potential fluctuations is so great that their averaging makes the potentials appear much smoother. As is generally the case for records taken from excellent preparations, light adaptation reversibly hyperpolarizes the cell (see Easland \& Wasserman, 1979). The magnitude of the hyperpolarization is indicated by the horizontal dashed lines that intersect the light-adapted responses; they represent the dark-adapted baseline potential.

These responses are quantified in Figure 3, which plots intensity-response functions for the transient peak of the response and for the steady-state plateau of the response in both dark and light adaptation. To avoid the paradox that the light-adapted responses would be bigger than the dark-adapted responses if all responses were measured in relative units with respect to their own baselines, all responses have been measured relative to an absolute

Figure 2 (opposite page). Receptor potentials evoked in single Limulus photoreceptor cells in dark and light adaptation (DA and LA) as a function of the intensity of a 5,120-msec light-flash. Flash duration is indicated by the horizontal line at the bottom of each column of responses. Intensity in $\log$ units of attenuation relative to a flash of $245 \mathrm{~W} / \mathrm{m}^{2}$ is given to the left of each receptor potential. Adaptation was regulated by presenting a 40-msec light-flash at 30-sec intervals. In DA, the adapting flash was attenuated by 3.9 log units; in LA, by 0.9 units. Penetrated cells that last for a long time while maintaining a stable sensitivity are generally hyperpolarized by light adaptation (see Easland \& Wasserman, 1979). The LA responses have therefore been positioned vertically with respect to their DA counterparts in order to show the extent of this hyperpolarization. The potential level of the DA baseline (the membrane potential) is indicated in relation to the LA responses by a horizontal dashed line. Quantum fluctuations caused by stray light sometimes occurred before flash onset, leading to an irregular preflash baseline; this effect is more apparent in DA. Recordings were made in the daytime from mature animals kept on a 12:12-h light:dark cycle. 

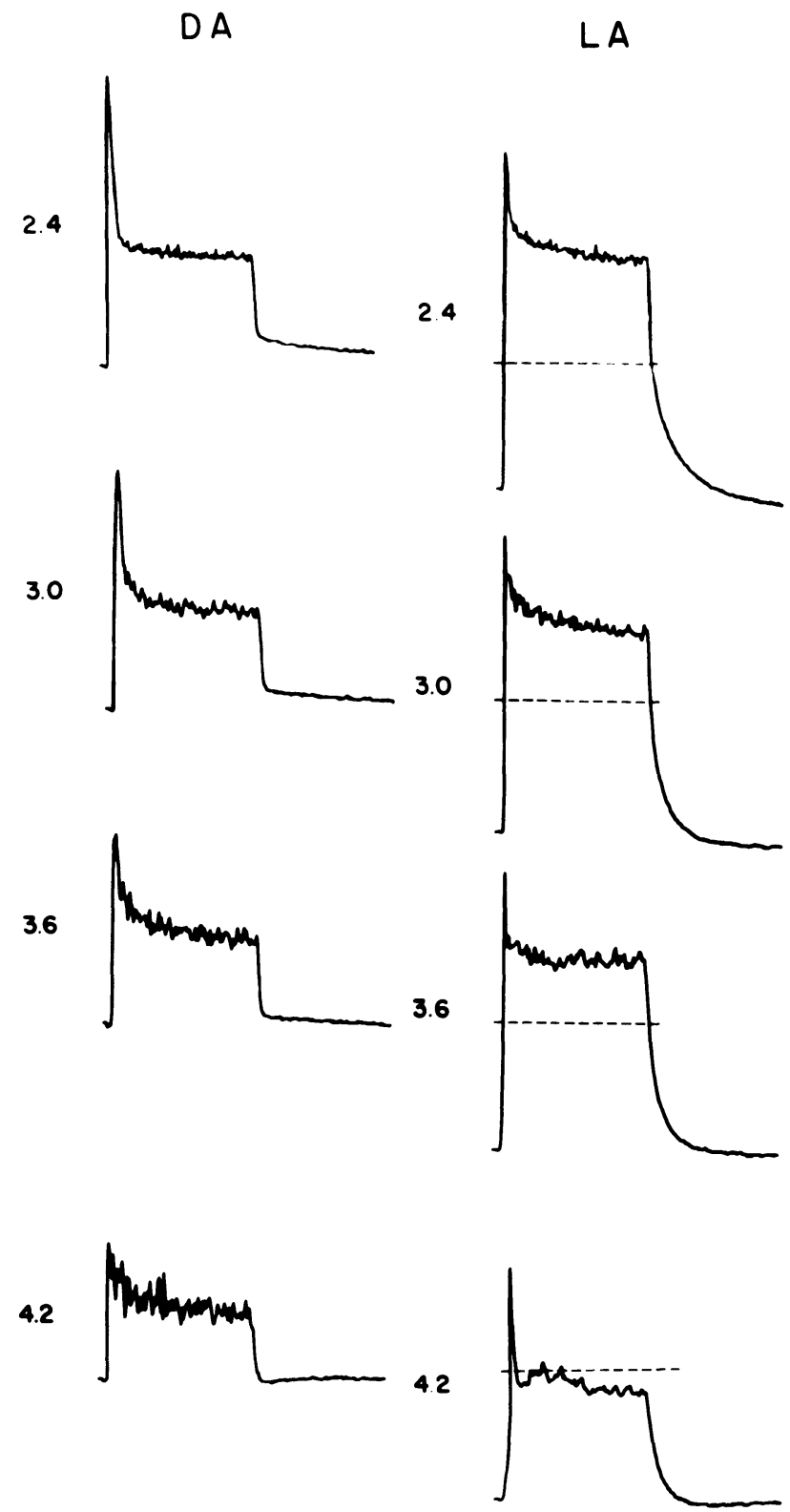

$48 \int$ Hormand
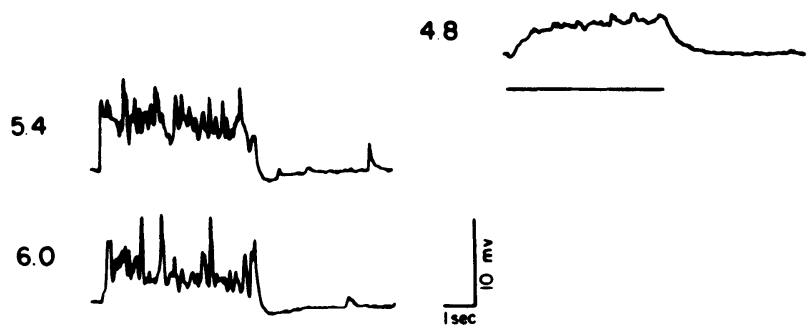


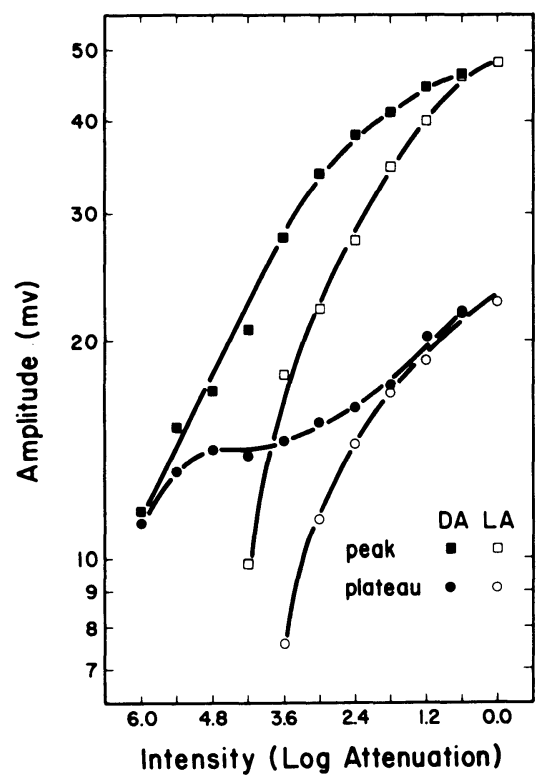

Figure 3. Intensity-response functions of excised Limulus lateral eye photoreceptor cells in dark and light adaptation (DA and LA). The data points for the peak represent the amplitude of the early transient response. The plateau data points represent the average depolarization produced during the last $2 \mathrm{sec}$ of the response. All responses were measured absolutely with respect to the DA baseline.

potential level-specifically, the dark-adapted baseline. Light-adapted responses that never intersected this absolute reference point (compare the $-4.8 \mathrm{log}$ attenuation unit response in light-adaptation in Figure 2) therefore do not appear on the logarithmic response coordinate used in Figure 3.

The functions in Figure 3 converge in several places. These superficially striking convergences are actually trivial expressions of the presence or absence of the rapid self-adaptation that the response itself may produce. Thus, at low stimulus intensities, where little self-adaptation occurs, the peak and plateau functions necessarily converge because there is no sag from peak to plateau. Conversely, at high intensities, where the self-adaptation produced by the test flash on its own response is much more powerful than the differential adaptation produced by the train of adapting flashes, the dark- and light-adapted plateau functions necessarily converge.

Of particular importance in the present case is the duality evident in Figure 3 in the intensity-response function for the dark-adapted plateau; it rises at low intensities, then levels off, and then rises yet again at high intensities. Functions of this form taken from intact eyes were interpreted by Barlow and his co-workers as evidence for the existence of two mechanisms operating at different levels of sensitivity. Excision was believed to abolish the more sensitive mechanism. But Figure 3 clearly shows the existence of two segments of the intensity-response function in excised eyes. It also shows that light adapta- tion in excised eyes eliminates the more sensitive segment of this function and dark adaptation restores it.

It should be noted that the segmented appearance of the dark-adapted plateau function may be only a trivial consequence of the set of function convergences that automatically follow from the fact of self-adaptation: Given that the dark-adapted plateau function must converge at low intensities with the dark-adapted peak function and that it must also converge at high intensities with the lightadapted plateau function, and given further that the greater the difference in sensitivity induced by changing the adaptation state, the greater will be the separation between the dark-adapted peak function and the light-adapted plateau function, it is possible that the relatively flat intermediate section of the dark-adapted plateau function is merely a secondary consequence of the fact that this function is monotonic and continuous. Thus, these data may not indicate any duality of mechanism, only a duality of appearances.

Finally, we suggest that the main influence of the preparation type is on the work habits of the investigators. An intact organism will be expected to last for long periods, thereby permitting a more leisurely research design. An excised preparation cannot be expected to last indefinitely. In this case, investigators will be less likely to allow the time needed for full dark adaptation. But when the exigencies of the work itself require that one only examine cells that are stable for hours after penetration, the difference between intact and excised preparations seems less than compelling.

\section{REFERENCES}

ADOLPH, A. R. (1964). Spontaneous slow potential fluctuations in the Limulus photoreceptor. Journal of General Physiology, 48, 297-322. Barlow, R. B., JR., \& Kaplan, E. (1971). Limulus lateral eye: Properties of receptor units in the unexcised eye. Science, 174, 1027-1029.

Barlow, R. B., JR., \& Kaplan, E. (1977). Properties of visual cells in the lateral eye of Limulus in situ: Intracellular recordings. Journal of General Physiology, 69, 203-220.

BAYER, D. S., \& BARLOW, R. B., JR. (1978). Limulus ventral eye: Physiological properties of photoreceptor cells in an organ culture medium. Journal of General Physiology, 72, 539-563.

Dowling, J. E. (1968). Discrete potentials in the dark-adapted eye of the crab Limulus. Nature, 217, 28-31.

EASLAND, G., \& Wasserman, G. S. (1979). Multiple intracellular contributions to light adaptation in Limulus ommatidia. Vision Research, 19, 1-8.

Fuortes, M. G. F., \& Yeandle, S. (1964). Probability of occurrence of discrete potential waves in the eye of Limulus. Journal of General Physiology, 47, 443-463.

Kaplan, E., \& Barlow, R. B., JR. (1975). Properties of visual cells in the lateral eye of Limulus in situ: Extracellular recordings. Journal of General Physiology, 66, 303-326.

WANG, L. T., \& WASSERMAN, G. S. (1980). Effect of adaptation on photoreceptor response duality in Limulus. Society for Neuroscience Abstracts, 6, 194.

WANG, L. T., \& Wasserman, G. S. (1984). Visual cell properties in excised Limulus eyes. Manuscript submitted for publication.

WANG, L. T., \& WASSERMAN, G. S. (1985). Direct intracellular measurement of nonlinear postreceptor transfer functions in dark and light adaptation in Limulus. Brain Research, 328, 41-50.

(Manuscript received July 29, 1990.) 